\title{
Hepatitis B Reactivation in a Bone Marrow Transplanted Patient: A Case Report (Hepatitis B Reactivation)
}

\author{
Ahmet Uyanikoglu* \\ Medical Faculty, Gastroenterology, Harran University, Sanliurfa, Turkey \\ *Corresponding Author: Ahmet Uyanıkoglu, Medical Faculty, Gastroenterology, Harran University, Sanliurfa, Turkey.
}

Received: August 06, 2019; Published: September 23, 2019

DOI: 10.31080/ASGIS.2019.02.0081

\begin{abstract}
HBsAg positive patients with hematological malignancies have a high risk of developing hepatitis B virus (HBV) reactivation when using chemotherapy agents and bone marrow transplantation (BMT). HBV reactivation has been reported in lymphoma patients with HBsAg-negative, anti-HBc IgG and anti-HBs-positive (resolved HBV infection) when BMT was used. A middle-aged female patient with HBV reactivation after BMT is presented.

Keywords: Hepatitis B Reactivation; Lymphoma; BMT
\end{abstract}

\section{Introduction}

HBsAg positive patients with hematological malignancies have a high risk of developing hepatitis B virus (HBV) reactivation when using high-dose corticosteroids, rituximab, anthracycline group chemotherapy agents and bone marrow transplantation (BMT) [1]. In the literature, HBV reactivation has been reported in lymphoma patients with hepatitis B surface antigen (HBsAg)-negative, antibody to hepatitis B core antigen (anti-HBc IgG) and antibody to hepatitis B surface antigen (anti-HBs)-positive (resolved HBV infection) lymphoma when BMT is used or rituximab as a chemotherapeutic agent [2-4].

All patients with hematological malignancies receiving anticancer therapy should be screened for active or resolved HBV infection by blood tests for HBsAg and anti-HBc IgG. Patients found to be positive for HBsAg should be given prophylactic antiviral therapy to prevent HBV reactivation. For patients with resolved HBV infection, no standard strategy has yet been established to prevent HBV reactivation [1].

A middle-aged female patient with HBV reactivation after BMT is presented.

\section{Case Report}

The 43-year-old woman presented to the gastroenterology clinic with complaints of fatigue, weight loss and jaundice. In her history, it was learned that she had been diagnosed with hodgkin's lymphoma due to ebstein barr virus (EBV) and BMT was usedseven months ago. Her family history was unremarkable. On physical examination, fever was $37 \mathrm{C}$, pulse was 90 beats/minute, blood pressure was 110/75 mm Hg, SPO2 98\% and she was icteric. In the abdominal examination, there was no defensive rebound, the right upper quadrant was sensitive to deep palpation. In pre-BMT studies, HBsAg-negative, ant-HBc IgG-positive, anti-HBs-positive, anti-HCV-negative, ALT and AST were within normal limits. The patient was admitted to the gastroenterology service. Biochemistry: AST: 356 U/L, ALT: 590 U/L, ALP: 291 U/L, GGT: 293 U/L, total bilirubin: $8.5 \mathrm{mg} / \mathrm{dl}$. Viral serology HBsAg-positive, anti-HBc IgMpositive, anti-HBc IgG-positive was detected. The patient was diagnosed with acute hepatitis B reactivation after BMT and entecavir $0.5 \mathrm{mg}$ was started. Since the 3rd day of the treatment, the clinical condition began to improve, and tranaminases and bilirubin levels regressed. The patient was discharged from hospital on the first week of her admission.

\section{Discussion}

HBV reactivation may occur in $>10 \%$ of patients with lymphoma and resolved HBV infection who undergo rituximab-containing chemotherapy. Preventive strategies may have marked impact on resource allocation in HBV endemic areas [5]. Our country is moderate endemic area [6]. On the otherhand the role of antiviral prophylaxis before chemotherapy or immunosuppressive therapy to prevent $\mathrm{HBV}$ reactivation in patients with resolved HBV infection is unclear. Our patient was in pre-BMT studies, HBsAg-negative, antHBc IgG-positive, anti-HBs-positive, and didn't started prophylaxis before chemotherapy and BMT.

Acute HBV infection and acute exacerbations of chronic HBV infection can cause acute liver injury or fulminant hepatitis. At this stage, spontaneous survival is poor, less than $25 \%$ [7]. Our patient was acute hepatitis B, on resolved hepatititis B. She hadn't fulminant hepatitis, entecavir was started. Since the 3rd day of the treatment, the clinical condition began to improve. 
In the literature, negative anti-HBs status and rituximab-containing regimens are both important factors for predicting chemotherapy or immunosuppressive therapy-related HBV reactivation in patients with resolved HBV infection. Therefore, antiviral prophylaxis should be considered in this patient population [2,3]. In our patient anti-HBs was positive, didn't given prophylactic antiviral theraphy and was seen HBV reactivation.

HBV reactivation is a serious complication for patients with lymphoma treated with rituximab-containing chemotherapies, despite lamivudine prophylaxis treatment. An optimal prophylactic antiviral protocol has not been determined.Among patients seropositive for the hepatitis B surface antigen with diffuse large B-cell lymphoma undergoing chemotherapy, the addition of entecavir compared with lamivudine resulted in a lower incidence of HBVrelated hepatitis and HBV reactivation. These findings support the use of entecavir in these patients [8]. Entecavir or tenofovir are preferred over lamivudine as prophylactic therapy [1]. Entecavir was started, since the 3rd day of the treatment, the clinical condition began to improve in our patient.

Organ transplantation is a lifesaving procedure for many patients. To prevent rejection or graft-versus-host disease, recipients require long-term immunosuppression. In patients who have ever been exposed to hepatitis $B$, it is possible for reactivation to occur; this includes patients who are anti-HBc IgG-positive only or both anti-HBc IgG-positive and anti-HBs-positive [9].

Patients with malignancies require chemotherapy and other immunosuppressive therapies for treatment. Because of this immunosuppression, in patients who have ever been exposed to hepatitis B it is possible for reactivation to occur. This reactivation can be fatal. Reactivation is particularly likely in patients who receive $B$ cell-active agents such as rituximab. The occurrence of reactivation flares may also delay further chemotherapy, which can negatively affect the outcome of the underlying malignancy. Accordingly, it is important to screen patients for markers of hepatitis B and institute antiviral prophylaxis to prevent reactivation [10].

\section{Conclusion}

Even if the anti-HBs is positive after resolved hepatitis B infection, acute hepatitis $B$ reactivation may be seen in patients who underwent BMT if the oral antiviral prophylaxis is neglected as in our patient. If such a situation is encountered, rescue therapy should be performed with strong antivirals such as entecavir or tenofovir and close monitoring until at least 6 months after anticancer therapy is required.

\section{Bibliography}

1. Law MF., et al. "Prevention and management of hepatitis B virus reactivation in patients with hematological malignancies treated with anticancer therapy". World Journal of Gastroenterology 22.28 (2016): 6484-500.
2. Su YC., et al. "Hepatitis B virus reactivation in patients with resolved hepatitis B virus infection receiving chemotherapy or immunosuppressive therapy". European Journal of Gastroenterology and Hepatology 30.8 (2018): 925-929.

3. Cho Y., et al. "High titers of anti-HBs prevent rituximab-related viral reactivation in resolved hepatitis $B$ patient with non-Hodgkin's lymphoma”. Journal of Medical Virology 88.6 (2016):1010-1017.

4. Yeo W., et al. "Hepatitis B virus reactivation in lymphoma patients with prior resolved hepatitis B undergoing anticancer therapy with or without rituximab". Journal of Clinical Oncology 27.4 (2009): 605-611.

5. Tsou HH., et al. "Taiwan Cooperative Oncology Group. Costeffectiveness of preventing hepatitis $B$ virus reactivation in patients with lymphoma and resolved HBV infection". Journal of the Formosan Medical Association (2019).

6. Uyanikoglu A. “Chronic Hepatitis B Infection”. Journal of Infectious Diseases and Therapy 2 (2014): 2.

7. Ichai P and Samuel D. "Management of Fulminant Hepatitis B". Current Infectious Disease Reports 21.7 (2019): 25.

8. Huang H., et al. "Entecavir vs lamivudine for prevention of hepatitis B virus reactivation among patients with untreated diffuse large B-cell lymphoma receiving R-CHOP chemotherapy: a randomized clinical trial". The Journal of the American Medical Association 12.23 (2014): 2521-2530.

9. Sasadeusz J., et al. "Screening and Prophylaxis to Prevent Hepatitis B Reactivation: Transplant Recipients". Clinical Liver Disease 23.3 (2019): 493-509.

10. Sasadeusz J., et al. "Screening and Prophylaxis to Prevent Hepatitis B Reactivation: Patients with Hematological and Solid Tumor Malignancies". Clinical Liver Disease 23.3 (2019): 511519.

\section{Volume 2 Issue 8 October 2019 (c) All rights are reserved by Ahmet Uyanikoglu.}

